\title{
Hyperkeratotic porokeratosis ptychotropica with satellite lesions: a rare presentation of an unusual variant of porokeratosis
}

\author{
Debeeka Hazarika1, Manoj Pawar ${ }^{\circledR}$
}

\begin{abstract}
Since its description in 1995, porokeratosis ptychotropica (PP) has remained a less-recognized variant of porokeratosis (PK). The term porokeratosis ptychotropica was coined in reference to its characteristic of affecting body folds. It mimics many other dermatological diseases and is therefore often misdiagnosed. We report a patient with multiple hyperkeratotic, warty lesions across the buttocks that mimicked cutaneous tuberculosis (CTB), but histological examination confirmed the correct diagnosis of PP.
\end{abstract}

Keywords: porokeratosis ptychotropica, cornoid lamella

Received: 12 May 2017 | Returned for modification: 18 May 2017 | Accepted: 24 May 2017

\section{Introduction}

Porokeratosis (PK) is an uncommon disorder of epidermal keratinization characterized by the appearance of one or more atrophic patches surrounded by a unique ridge-like border, histologically termed a cornoid lamella (1). Porokeratosis ptychotropica (PP) is a lesser-known variant of PK characterized by verrucous plaques that are often pruritic and commonly involve the gluteal cleft with or without the extremities (2).

\section{Case report}

A 40-year-old otherwise healthy Indian housewife presented with grayish warty lesions across both buttocks that had persisted for 1 year. A year earlier, she had developed an asymptomatic small elevated skin-colored lesion on the left buttock that had increased in size and spread centrifugally with mild flattening of the center, attaining its present size, and similar lesions of varying sizes also developed in the vicinity of and across the right buttock. There was no history of trauma, insect bite, or any drug intake prior to the onset of the initial lesion (Fig. 1). The patient noted itching and discomfort in the affected region. Her medical history and family history were insignificant. She had previously been treated with topical steroids, retinoids, and electrocauterization at various other centers, with a minimal response. All relevant laboratory investigations were normal.

Examination revealed two well-demarcated, brownish, annular, verrucous plaques: one measuring $6 \times 7 \mathrm{~cm}$ with raised borders and a depressed center across the left buttock, and one measuring $3 \times 4 \mathrm{~cm}$ across the right buttock, without central atrophy and surrounded by multiple satellite hyper-pigmented papules and plaques.

A skin biopsy of the larger lesion was obtained. Histological examination showed multiple, keratin-filled invagination, a parakeratotic column (i.e., cornoid lamella), a loss of the granular layer of the underlying epidermis, and dense lympho-histiocytic infiltrate in the dermis, compatible with porokeratosis (Fig. 2). Congo red staining for amyloid deposits was negative.

Based on typical clinical and histological examination, the patient was diagnosed as a case of the hyperkeratotic variant of PP.
Cryotherapy was performed on the lesions, resulting in clearing of the smaller lesions. However, the larger lesions persisted, and so she was referred to plastic surgery for total excision of these.

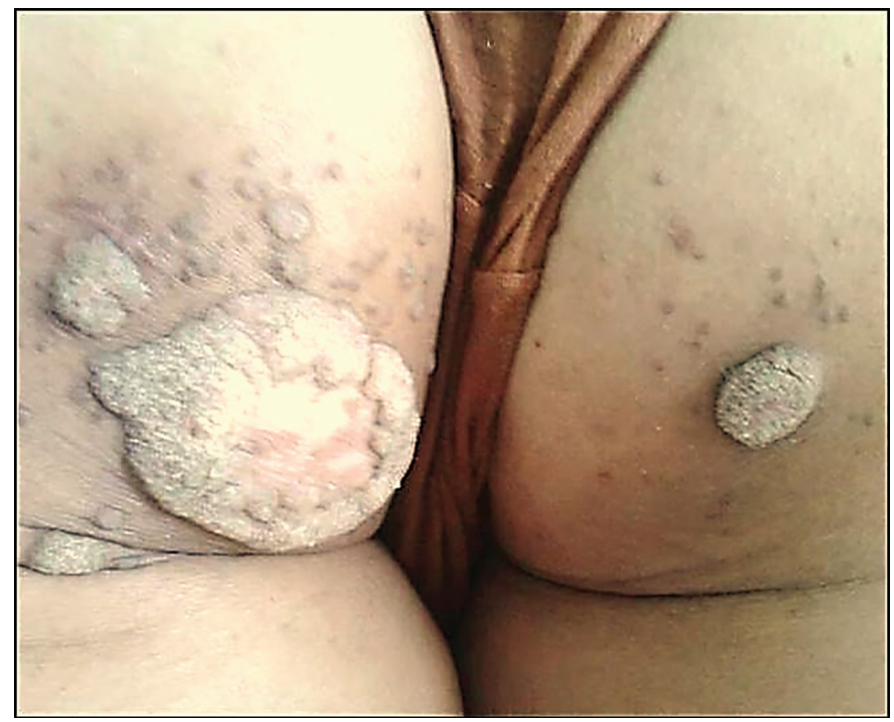

Figure 1 | A well-defined, hyperkeratotic annular plaque measuring $6 \times 7 \mathrm{~cm}$ with depressed center across the left buttock with hyperpigmented papules and plaques in the vicinity and a well-defined, hyperkeratotic annular plaque measuring $3 \times 4 \mathrm{~cm}$ size across the right buttock with satellite papules.

\section{Discussion}

PK is classified as porokeratosis of Mibelli (PM), disseminated superficial porokeratosis of immunosuppression and childhood, disseminated superficial actinic porokeratosis (DSAP), linear and punctate forms, porokeratosis palmaris et plantaris disseminate, giant PK, and less common PP. Various triggers for all forms of PK include infections, drugs, ultraviolet radiation, trauma and immunodeficiency resulting from organ transplantation, and/or hematologic malignancies (1-3), which result in abnormal clonal hyperproliferation of atypical keratinocytes with centrifugal spread, resulting in characteristic cornoid lamella in histology (1).

PP was initially described in a 34-year-old man by Lucker et al. in 1995 (2). The term porokeratosis ptychotropica is derived from the Greek words ptyche 'fold' and trope 'turning'. It is a symmetric eruption, consisting of erythematous papules and plaques localized 


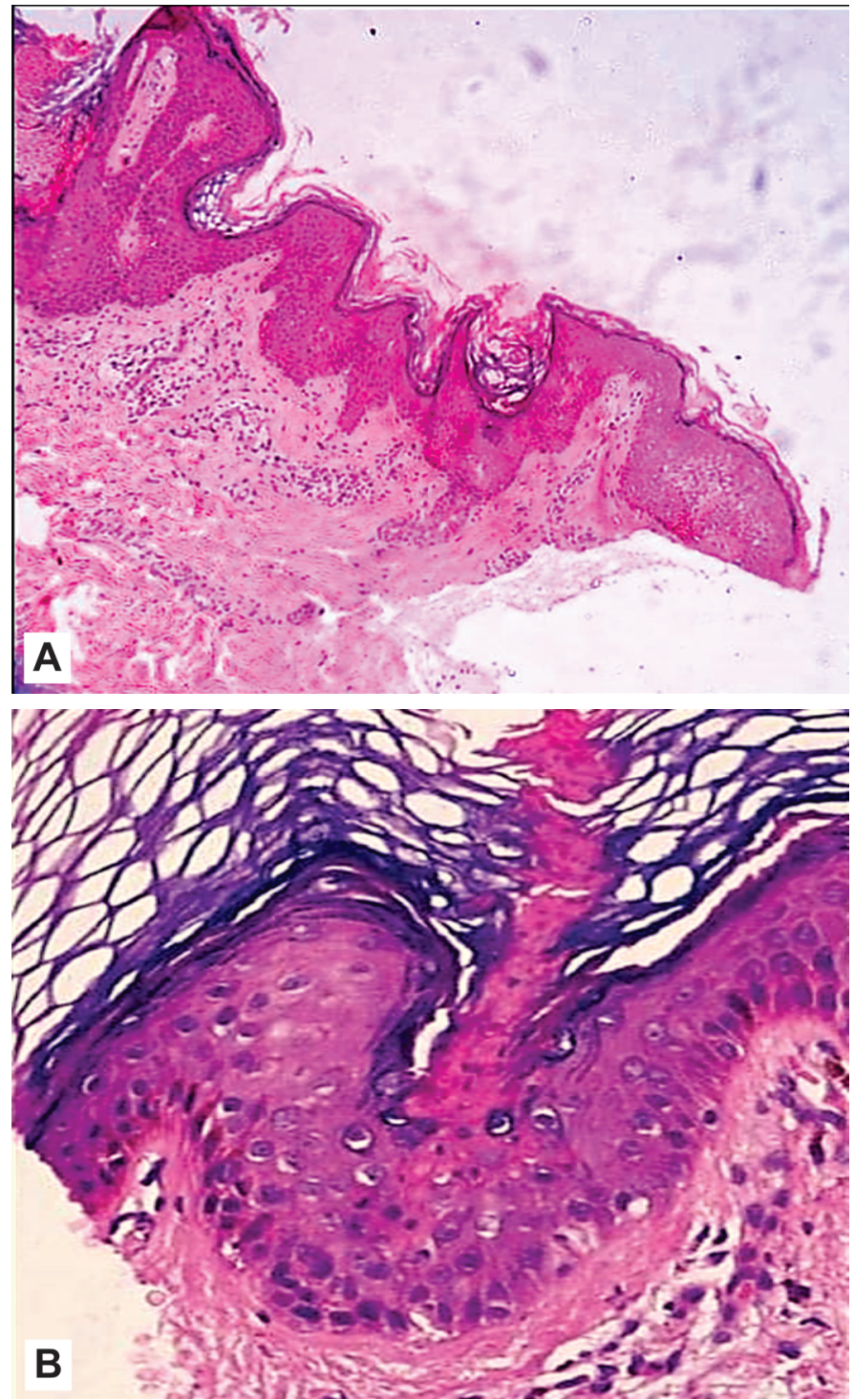

Figure 2 | A) Histology showing multiple, keratin-filled invagination, a parakeratotic column (i.e., cornoid lamella), loss of the granular layer of underlying epidermis, and dense lympho-histiocytic infiltrate in the dermis (H\&E, 40×). B) The same histology at higher magnification (H\&E, 100x).

to the perianal and gluteal region that occasionally show regional extension to the thighs and genitals. These lesions have a tendency to coalesce centrally and expand peripherally. It is quite common in men and pruritus often occurs with it (1-4).

The classic lesion of PP resembles inverse psoriasis, verruca vulgaris, chronic intertrigo, chronic contact/irritant dermatitis, acrodermatitis enteropathica, and necrolytic migratory erythema (3). Interestingly, in our patient the lesions were large, hyperkeratotic, warty plaques with multiple similar but smaller lesions in the vicinity, which resembled tuberculosis verrucosa cutis and/ or warty lupus vulgaris, and this kind of unique presentation of PP is less well known (5) because most reported cases of PP have resembled either psoriasis or verruca vulgaris $(4,6-8)$. Cutaneous tuberculosis (CTB) is not uncommon in India; its overall prevalence varies from 0.25 to $0.6 \%$ and the buttocks are more often involved sites than the face (9). Hence, our initial differential diagnoses were CTB (specifically, tuberculosis verrucosa cutis and the plaque form of lupus vulgaris), and we also considered giant warts and verrucous squamous cell carcinoma (SCC) as differentials, but definitive histology confirmed the correct diagnosis. Histological examination of PP characteristically shows psoriasiform hyperplasia and multiple cornoid lamellae, which probably result in the hyperkeratotic and/or verruciform nature of the plaques and also distinguish it from other forms of PK. In many cases of PP there was amyloid deposition in the papillary dermis $(4,6-8)$. Chronic inflammation, pruritus, and friction leading to basal layer degeneration beneath the cornoid lamellae may lead to these deposits (8).

PK patients often have underlying immune suppression; however, to date there has been no such association reported with PP $(3,6,10)$. All variants of porokertaosis, except punctate, are prone to malignant transformation; for example, into Bowen's disease, SCC, and rarely basal cell carcinoma, the risk of which varies from 7.5 to $11.6 \%(1,10)$. Mazori et al. noted the development of invasive SCC in PP lesions and they speculated that the down-regulation of p16 may have played a role in it (10).

Treatment options for PP depend on the size and location of the lesion, functional and aesthetic considerations, and the patient's condition, but it is often refractory to therapy. Topical keratolytics, 5-flurouracil, corticosteroids, imiquimod, Vit.D analogues, oral and topical retinoids, podophyllin, intralesional bleomycin, electrocautery, dermabrasion, cryosurgery, $\mathrm{CO} 2$ laser, and wide surgical excision are various treatment modalities that yield only symptomatic improvement without affecting the lesion itself much (1-3).

Clinicians should be aware of such a rare, pruritic condition across the buttocks with atypical presentation and should consider porokeratosis ptychotropica in differentials, especially in patients from geographical areas where the prevalence of CTB is not low. Early detection and long-term follow-up is necessary because these lesions are prone to malignant changes.

\section{References}

1. Oji V, Metze D, Traupe H. Inherited disorders of cornification. In: Griffiths C, Barker J, Bleiker T, Chalmers R, Creamer D. Rook's textbook of dermatology. 9th ed. Chichester, UK: Wiley Blackwell; 2016: Chapter 65, p. 67-8.

2. Lucker GP, Happle R, Steijlen PM. An unusual case of porokeratosis involving the natal cleft: porokeratosis ptychotropica? Br J Dermatol. 1995;132:150-1.

3. Yeo J, Winhoven S, Tallon B. Porokeratosis ptychotropica: a rare and evolving variant of porokeratosis. J Cutan Pathol. 2013;40:1042-7.

4. Takiguchi RH, White KP, White CR Jr, Simpson EL. Verrucous porokeratosis of the gluteal cleft (porokeratosis ptychotropica): a rare disorder easily misdiagnosed. J Cutan Pathol. 2010;37:802-7.

5. Thomas C, Ogboli MI, Carr RA, Charles-Holmes R. Hypertrophic perianal porokeratosis in association with superficial actinic porokeratosis of the leg. Clin Exp Dermatol. 2003;28:676-7.

6. Lee DK, Oh SH, Chang SE, LEE MW, Choi JH, Moon KC, et al. A rare variant of porokeratosis: porokeratosis ptychotropica. J Am Acad Dermatol. 2006;55(Suppl): S120-2.
7. Jee MS, Chang SE, Suh HS, Choi JH, Sung KJ, Moon KC, et al. Porokeratosis ptychotropica associated with dermal amyloid deposits. Clin Exp Dermatol. 2003;28:450-2.

8. Tallon B, Blumental G, Bhawan J. Porokeratosis ptychotropica: a lesser-known variant. Clin Exp Dermatol. 2009;34:e895-7.

9. Pai VV, Naveen KN, Athanikar SB, Dinesh US, Divyashree A, Gupta G. A clinicohistopathological study of lupus vulgaris: a 3 year experience at a tertiary care centre. Indian Dermatol Online J. 2014;5:461-5.

10. Mazori DR, Shvartsbeyn M, Meehan SA, Tarsis SL. Transformation of porokeratosis ptychotropica into invasive squamous cell carcinoma. Int J Dermatol. 2017;56:679-80. 